\title{
Effect of Different Dose of NPK on Flower Phenology of Dragon Fruit
}

\author{
Tamanna Perween* and M.A. Hasan \\ Department of Fruits and Orchard Management, Bidhan Chandra Krishi Viswavidyalaya, \\ Mohanpur, Nadia, 741252, West Bengal, India \\ *Corresponding author
}

\begin{abstract}
A B S T R A C T
An investigation was carried out with different doses of NPK nutrients (viz. $\mathrm{T}_{1}-\mathrm{N}_{250} \mathrm{P}_{2} \mathrm{O}_{5}$ ${ }_{150} \mathrm{~K}_{2} \mathrm{O}_{100} \mathrm{~g} /$ pole; $\mathrm{T}_{2}-\mathrm{N}_{300} \mathrm{P}_{2} \mathrm{O}_{5} 200$ K2 $\mathrm{O}_{150} \mathrm{~g} /$ pole; $\mathrm{T}_{3}-\mathrm{N}_{350} \mathrm{P}_{2} \mathrm{O}_{5}{ }_{250} \mathrm{~K}_{2} \mathrm{O}_{200} \mathrm{~g} /$ pole; $\mathrm{T}_{4}-\mathrm{N}$

\section{Keywords}

Dragon Fruit, Flower morphology, Nutrient, NPK, Phenology

Article Info

Accepted:

18 April 2018 Available Online:

10 May 2018 $400 \mathrm{P}_{2} \mathrm{O}_{5} 300 \mathrm{~K}_{2} \mathrm{O} 250$ g/pole; $\mathrm{T}_{5}-\mathrm{N}_{450} \mathrm{P}_{2} \mathrm{O}_{5350} \mathrm{~K}_{2} \mathrm{O}_{300} \mathrm{~g} /$ pole; $\mathrm{T}_{6}-\mathrm{N}_{500} \mathrm{P}_{2} \mathrm{O}_{5400} \mathrm{~K}_{2} \mathrm{O} 350$ g/pole; $\mathrm{T}_{7}-\mathrm{N}_{550} \mathrm{P}_{2} \mathrm{O}_{5450} \mathrm{~K}_{2} \mathrm{O}_{400} \mathrm{~g} /$ pole and $\mathrm{T}_{8}-\mathrm{Control}$ ) in premises adjacent to the Faculty of Horticulture, Bidhan Chandra Krishi Viswavidyalaya, Mohanpur, Nadia, West Bengal, India during the years 2015 and 2016 to evaluate the effects of NPK nutrients on flower morphology of dragon fruit (Hylocereus costaricensis) cv. Royal Moroccan Red.The experiment was laid out in a randomised block design with 8 (eight) treatments and 3 (three) replications. Each treatment comprised of one pole having 4 (four) plants. The observations were recorded on days to first flower, flower length, androecium characters, gynoecium characters and different parts of perianth was observed. The days required to first flower was 431.25 days with application of $\mathrm{N}_{350} \mathrm{P}_{2} \mathrm{O}_{5250} \mathrm{~K}_{2} \mathrm{O}_{200} \mathrm{~g} /$ pole and flowering became earlier by nearly 15 days over control. Among the different flower parameters, the length of upper, inner, outer and lower free perianth, style, stamen and the ovary were only significantly influenced due to the different treatments.
\end{abstract}

\section{Introduction}

Hylocereus costaricensis commonly called as Dragon fruit or Pithaya which is a native of Mexico and Central and South America (Britton and Rose, 1963; Mizrahi et al., 1997; Morton, 1987).

It is a long day plant with beautiful night blooming flower that is nicknamed as "Noble Woman" or "Queen of the Night". The fruit is also known as Strawberry Pear, Dragon fruit, Pithaya, Night blooming Cereus, Belle of the night, Conderella plant and Jesus in the Cradle. It is a long day plant usually flowering starts from April to November sometimes extending till December and occurs in four to six flushes and sometimes to seven (Perween et al., 2018). Pitahaya belongs to the genus Hylocereus $(2 \mathrm{n}=22)$. Young stems and fresh flower buds are eaten as vegetables, while dried ones are used for homemade medicine (Ortiz-Hernández, 1999).

In Taiwan, dry flowers are consumed as vegetables (Mizrahi and Nerd, 1999). Literature on morphology of dragon fruit lacking in general and on flower morphology in particular. There is no scientific information available in the India subcontinent on the 
effect of nutrient on flower morphology of dragon fruit. Hence the present investigation was undertaken to evaluate the response of NPK on flower morphology of dragon fruit.

\section{Materials and Methods}

The present study was conducted with eight fertilizer treatments viz., $\mathrm{T}_{1}=\mathrm{N}_{250} \mathrm{P}_{150} \mathrm{~K}_{100}, \mathrm{~T}_{2=}$ $\mathrm{N}_{300} \mathrm{P}_{200} \mathrm{~K}_{150}, \mathrm{~T}_{3}=\mathrm{N}_{350} \mathrm{P} 250 \mathrm{~K}_{200}, \mathrm{~T}_{4=} \mathrm{N}_{400} \mathrm{P}_{300}$ $\mathrm{K}_{250}, \mathrm{~T}_{5=} \mathrm{N}_{450} \mathrm{P}_{350} \mathrm{~K}_{300}, \mathrm{~T}_{6=} \mathrm{N}_{500} \mathrm{P}_{400} \mathrm{~K}_{350}, \mathrm{~T}_{7}=$ $\mathrm{N}_{550} \mathrm{P}_{450} \mathrm{~K}_{400}, \mathrm{~T}_{8}=$ Control and Organic manure @ $20 \mathrm{~kg} /$ pillar containing four plants was laid out in Randomized Block Design with 4 replications of NPK fertilizers combination at was conducted at premises of Faculty of Horticulture, Bidhan Chandra Krishi Viswavidyalaya, Mohanpur, Nadia, West Bengal, India during the period of 2015-2016.

The experimental field was situated at $23.5^{\circ} \mathrm{N}$ latitude and $89^{\circ} \mathrm{E}$ longitude on elevation 9.75 above mean sea level (MSL). Characters observed were days to first flower, flower length, androecium characters, gynoecium characters and different parts of perianths. Days to first flower was taken by days count from planting to first flower bloom of the plant.

All measurements were taken by slide caliper except length of flower taken by scale along with the perianth (upper, middle and lower) length. The data on different parameters were analyzed using analysis of variance (ANOVA) based on randomized block design (RBD).

\section{Results and Discussion}

The minimum days required to first flower was 431.25 in the treatment $\mathrm{T}_{3}\left(\mathrm{~N}_{350} \mathrm{P}_{2} \mathrm{O}_{5250}\right.$ $\mathrm{K}_{2} \mathrm{O}$ 200) which significantly differed with $\mathrm{T}_{2}$ (439.75 days) and maximum days (445.75) were required to first flowering in control $\left(\mathrm{T}_{8}\right)$. Highest stamen length of $11.25 \mathrm{~cm}$ was observed in $\mathrm{T}_{6}$ receiving $\mathrm{N}_{500} \mathrm{P}_{2} \mathrm{O}_{5} 400 \mathrm{~K}_{2} \mathrm{O}_{350}$ followed by $\mathrm{T}_{8}(11.03 \mathrm{~cm})$ and lowest stamen length was recorded in treatment $\mathrm{T}_{2}(9.99 \mathrm{~cm})$ which was at par with $\mathrm{T}_{7}(10.11 \mathrm{~cm})$. Ovary and style length varied significantly among the different treatments. The highest style length was observed in $\mathrm{T}_{2}(22.72 \mathrm{~cm})$ receiving $\mathrm{N}_{300}$ $\mathrm{P}_{2} \mathrm{O}_{5} 200 \mathrm{~K}_{2} \mathrm{O}_{150} \mathrm{~g} /$ pillar followed by in $\mathrm{T}_{7}$ $(21.99 \mathrm{~cm})$ and lowest in $\mathrm{T}_{4}(20.21 \mathrm{~cm})$ supplied with of $\mathrm{N}_{400} \mathrm{P}_{2} \mathrm{O}_{5300} \mathrm{~K}_{2} \mathrm{O}_{250}$. Among the treatments highest ovary length was recorded in treatment supplied with $\mathrm{N}_{450} \mathrm{P}_{2} \mathrm{O}_{5}$ $350 \mathrm{~K}_{2} \mathrm{O}_{300}(32.26 \mathrm{~cm})$ and lowest in control $(31.35 \mathrm{~cm})$. Perianth observed in pithaya flowers was hypothetically classified into four types:

a. Upper inner free perianth, b. Upper outer free perianth, c. Middle free perianth and $d$. Lower free perianth. Upper inner free perianth was free from the flower however, their lower part is indistinctly joined to the other flower parts. Length of upper free perianth varied significantly among the treatments with highest value of $13.42 \mathrm{~cm}$ in $\mathrm{T}_{2}\left(\mathrm{~N}_{300} \mathrm{P}_{2} \mathrm{O}_{5} 200\right.$ $\mathrm{K}_{2} \mathrm{O}_{150}$ ) and lowest upper inner free perianth length of $13.17 \mathrm{~cm}$ in $\mathrm{T}_{8}$ (control). Upper outer free perianth was similar to upper inner perianth but having green colour with reddish margin (Fig. 1 and 2).

Length of upper outer free perianth varied significantly among the treatments while number and diameter varied insignificantly. Highest upper outer free perianth length of $13.43 \mathrm{~cm}$ observed in $\mathrm{T}_{7}\left(\mathrm{~N}_{550} \mathrm{P}_{2} \mathrm{O}_{5}{ }_{450} \mathrm{~K}_{2} \mathrm{O}_{400}\right)$ and lowest in $\mathrm{T}_{5}(12.75 \mathrm{~cm})$. Middle free perianth was similar to upper outer perianth but smaller in size. Length of lower free perianth varied significantly among the treatments with highest value in the treatment $\mathrm{T}_{7}(33.72 \mathrm{~cm})$ and lowest in $\mathrm{T}_{1}(33.27 \mathrm{~cm})$. The unusually large, tubular style is $20 \mathrm{~cm}$ in length and $0.5 \mathrm{~cm}$ in diameter (Perween et al., 2018); the stigmas having 21 slender lobes, creamy green in colour (Daubresse Balayer et al., 1999; Luders, 1999; Perween et al., 2018) (Table 1 and 2). 
Table.1 Effect of nutrients on perianth of dragon fruit

\begin{tabular}{|c|c|c|c|c|c|c|c|c|c|c|c|c|}
\hline \multirow[b]{2}{*}{ Treatment } & \multicolumn{3}{|c|}{ Upper inner free perianth } & \multicolumn{3}{|c|}{ Upper outer free perianth } & \multicolumn{3}{|c|}{ Middle free perianth } & \multicolumn{3}{|c|}{ Lower free perianth } \\
\hline & Number & $\begin{array}{c}\text { Length } \\
(\mathrm{cm})\end{array}$ & $\begin{array}{c}\text { Diameter } \\
(\mathbf{m m})\end{array}$ & Number & $\begin{array}{c}\text { Length } \\
\text { (cm) }\end{array}$ & $\begin{array}{c}\text { Diameter } \\
(\mathbf{m m})\end{array}$ & Number & $\begin{array}{c}\text { Length } \\
\text { (cm) }\end{array}$ & $\begin{array}{c}\text { Diameter } \\
(\mathbf{m m})\end{array}$ & Number & $\begin{array}{c}\text { Length } \\
\text { (cm) }\end{array}$ & $\begin{array}{c}\text { Diameter } \\
(\mathbf{m m})\end{array}$ \\
\hline $\mathbf{T}_{1}$ & 10.50 & 13.20 & 13.35 & 11.50 & 13.24 & 13.16 & 9.00 & 8.52 & 13.06 & 31.25 & 33.27 & 13.06 \\
\hline $\mathbf{T}_{2}$ & 11.00 & 13.42 & 13.26 & 11.00 & 13.24 & 13.20 & 9.50 & 8.57 & 13.02 & 32.25 & 33.29 & 13.11 \\
\hline $\mathbf{T}_{3}$ & 11.25 & 13.25 & 13.22 & 11.50 & 13.22 & 13.21 & 9.75 & 8.51 & 13.04 & 31.50 & 33.33 & 13.05 \\
\hline $\mathrm{T}_{4}$ & 11.50 & 13.30 & 13.33 & 10.50 & 12.83 & 13.22 & 9.00 & 8.62 & 13.05 & 31.25 & 33.59 & 13.08 \\
\hline $\mathbf{T}_{5}$ & 11.50 & 13.20 & 13.29 & 10.75 & 12.75 & 13.21 & 10.00 & 8.63 & 13.04 & 31.50 & 33.53 & 13.03 \\
\hline$T_{6}$ & 11.50 & 13.30 & 13.27 & 11.25 & 13.10 & 13.23 & 9.50 & 8.61 & 13.09 & 31.25 & 33.28 & 13.15 \\
\hline$T_{7}$ & 11.00 & 13.37 & 13.26 & 11.25 & 13.43 & 13.19 & 9.00 & 8.56 & 13.09 & 31.50 & 33.72 & 13.06 \\
\hline$T_{8}$ & 11.00 & 13.18 & 13.28 & 12.50 & 13.20 & 13.19 & 9.00 & 8.51 & 13.06 & 31.25 & 33.28 & 13.03 \\
\hline $\mathrm{CD}_{0.05}$ & NS & 0.14 & NS & NS & 0.23 & NS & NS & NS & NS & NS & 0.15 & NS \\
\hline
\end{tabular}

Table.2 Effect of nutrients on gynoecium characters of dragon fruit

\begin{tabular}{|c|c|c|c|c|c|c|c|c|c|c|c|c|c|}
\hline Treatment & $\begin{array}{c}\text { Days } \\
\text { to } \\
\text { first } \\
\text { flower }\end{array}$ & $\begin{array}{l}\text { Flower } \\
\text { length } \\
\text { (cm) }\end{array}$ & $\begin{array}{c}\text { Petal } \\
\text { length } \\
\text { (cm) }\end{array}$ & $\begin{array}{c}\text { Petal } \\
\text { number }\end{array}$ & $\begin{array}{l}\text { Stamen } \\
\text { length } \\
\text { (cm) }\end{array}$ & $\begin{array}{l}\text { Anther } \\
\text { length } \\
\text { (mm) }\end{array}$ & $\begin{array}{l}\text { Anther } \\
\text { diameter } \\
(\mathrm{mm})\end{array}$ & $\begin{array}{c}\text { Style } \\
\text { length } \\
(\mathrm{cm})\end{array}$ & $\begin{array}{c}\text { Style } \\
\text { diameter } \\
(\mathbf{m m})\end{array}$ & $\begin{array}{c}\text { Number } \\
\text { of } \\
\text { stigma } \\
\text { lobes }\end{array}$ & $\begin{array}{l}\text { Stigma } \\
\text { lobe } \\
\text { length } \\
(\mathrm{mm})\end{array}$ & $\begin{array}{l}\text { Stigma } \\
\text { lobe } \\
\text { diameter } \\
(\mathbf{m m})\end{array}$ & $\begin{array}{c}\text { Ovary } \\
\text { length } \\
(\mathrm{mm})\end{array}$ \\
\hline $\mathrm{T}_{1}$ & 440.50 & 30.11 & 11.44 & 22.25 & 10.37 & 8.71 & 0.958 & 21.37 & 5.58 & 24.00 & 21.77 & 1.442 & 31.42 \\
\hline$T_{2}$ & 439.75 & 30.46 & 11.18 & 22.25 & 9.99 & 8.71 & 0.950 & 22.72 & 5.60 & 24.75 & 22.12 & 1.425 & 31.40 \\
\hline$T_{3}$ & 431.25 & 30.49 & 11.25 & 22.25 & 10.86 & 8.73 & 0.955 & 20.50 & 5.48 & 24.25 & 21.79 & 1.450 & 31.54 \\
\hline $\mathrm{T}_{4}$ & 440.75 & 30.62 & 11.17 & 21.75 & 10.45 & 8.72 & 0.958 & 20.21 & 5.63 & 23.75 & 21.51 & 1.435 & 31.52 \\
\hline$T_{5}$ & 441.25 & 30.77 & 11.39 & 22.00 & 10.64 & 8.70 & 0.948 & 20.41 & 5.48 & 24.50 & 22.21 & 1.433 & 32.26 \\
\hline$T_{6}$ & 441.25 & 31.07 & 11.27 & 21.50 & 11.25 & 8.71 & 0.955 & 20.28 & 5.63 & 24.50 & 21.62 & 1.430 & 31.39 \\
\hline $\mathbf{T}_{7}$ & 442.75 & 31.00 & 11.33 & 22.25 & 10.11 & 8.75 & 0.968 & 21.99 & 5.55 & 24.00 & 22.25 & 1.433 & 31.49 \\
\hline $\mathrm{T}_{8}$ & 445.75 & 30.05 & 11.28 & 22.00 & 11.03 & 8.73 & 0.950 & 21.55 & 5.55 & 24.25 & 21.73 & 1.423 & 31.35 \\
\hline $\mathrm{CD}_{0.05}$ & 6.7437 & NS & NS & NS & 0.5281 & NS & NS & 0.66 & NS & NS & NS & NS & 0.18 \\
\hline
\end{tabular}

NS- Non-significant

NOTE: $\mathrm{T}_{1}-\mathrm{N}_{250} \mathrm{P}_{2} \mathrm{O}_{5150} \mathrm{~K}_{2} \mathrm{O} 100 ; \mathrm{T}_{2}-\mathrm{N}_{300} \mathrm{P}_{2} \mathrm{O}_{5200} \mathrm{~K}_{2} \mathrm{O}_{150} ; \mathrm{T}_{3}-\mathrm{N}_{350} \mathrm{P}_{2} \mathrm{O}_{5250} \mathrm{~K}_{2} \mathrm{O}_{200} ; \mathrm{T}_{4}-\mathrm{N}_{400} \mathrm{P}_{2} \mathrm{O}_{5300} \mathrm{~K}_{2} \mathrm{O}_{250} ; \mathrm{T}_{5}-\mathrm{N}_{450} \mathrm{P}_{2} \mathrm{O}_{5350} \mathrm{~K}_{2} \mathrm{O}_{300} ; \mathrm{T}_{6}-\mathrm{N}_{500} \mathrm{P}_{2} \mathrm{O}_{5400} \mathrm{~K}_{2} \mathrm{O}$ ${ }_{350} ; \mathrm{T}_{7}-\mathrm{N}_{550} \mathrm{P}_{2} \mathrm{O}_{5450} \mathrm{~K}_{2} \mathrm{O}_{400} ; \mathrm{T}_{8}$ - Control. 
Fig.1 Different floral parts of dragon fruit flower

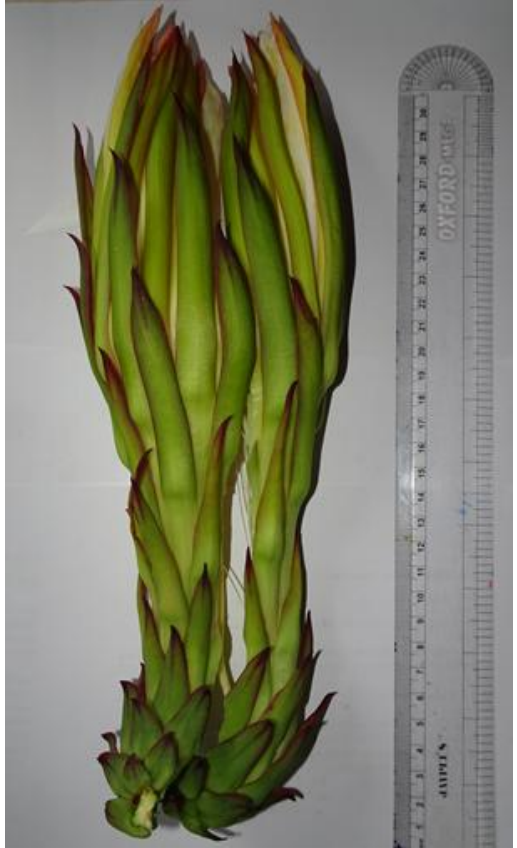

Cross section of flower (Dorsal view)

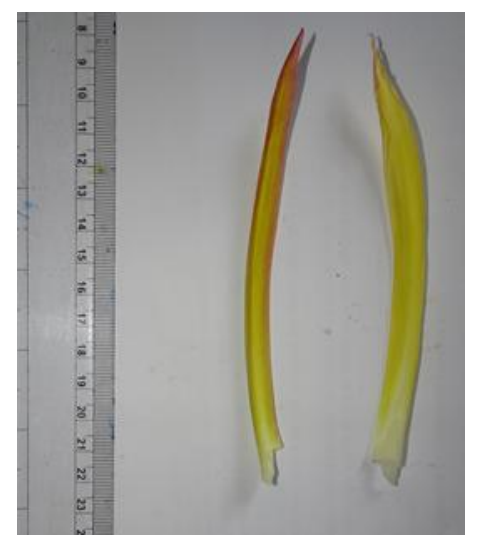

Upper inner perianth

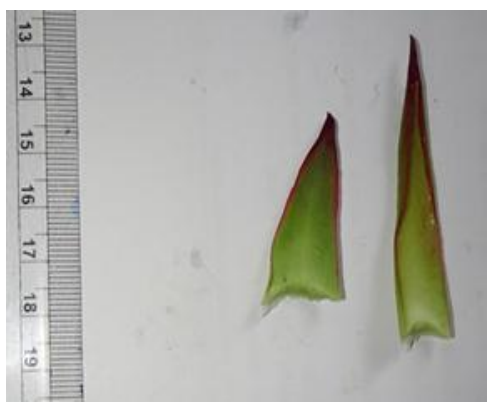

Lower perianth

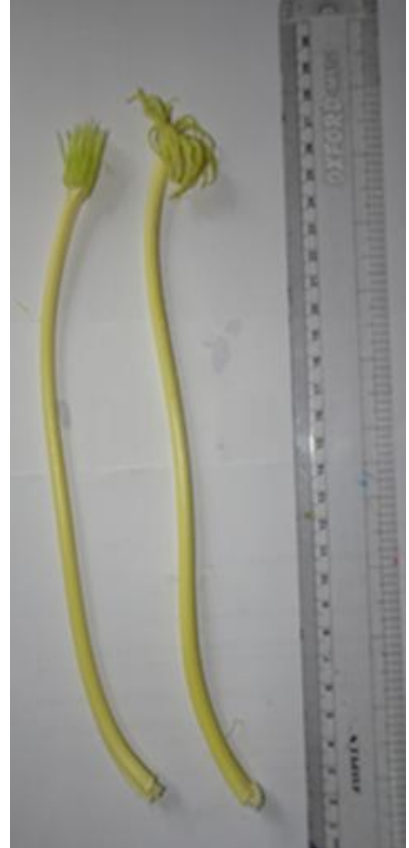

Style and Stigma lobes

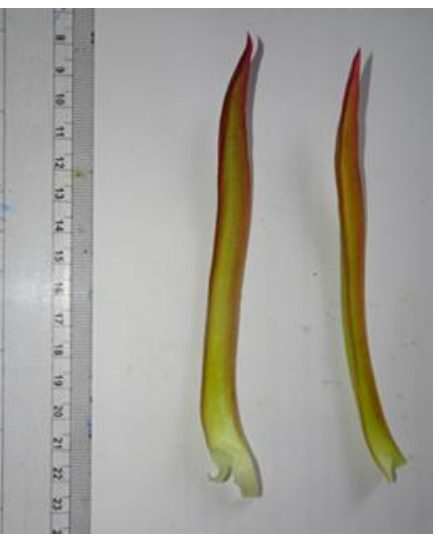

Upper outer perianth

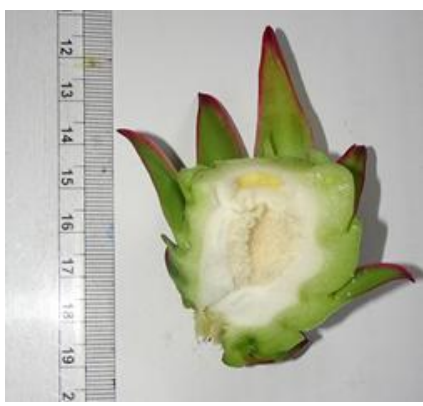

Ovary (cross section)

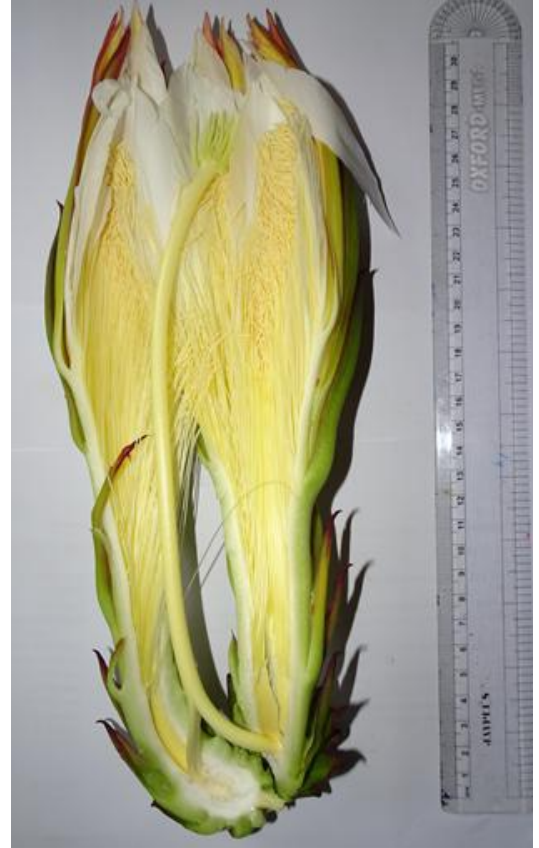

Cross section of flower (Ventral view)

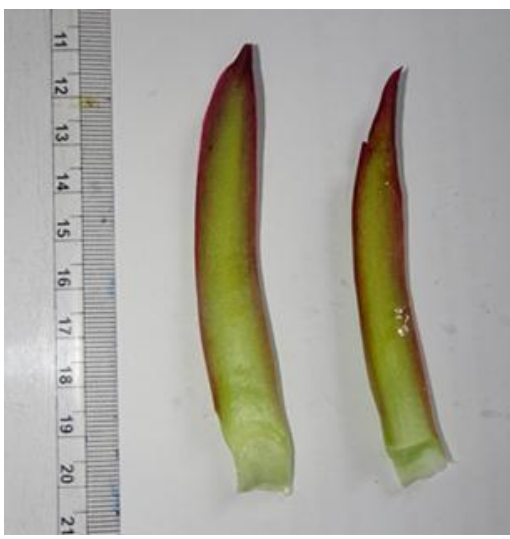

Middle perianth

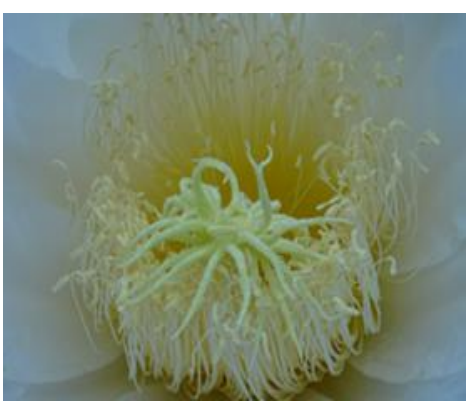

Stamens and stigma 
Fig.2 1- Flower bud initiation, 2, 3 and 4 - Flower buds, 5- Mature flower bud ready to bloom, 6 and 7 - Blooming of flowers
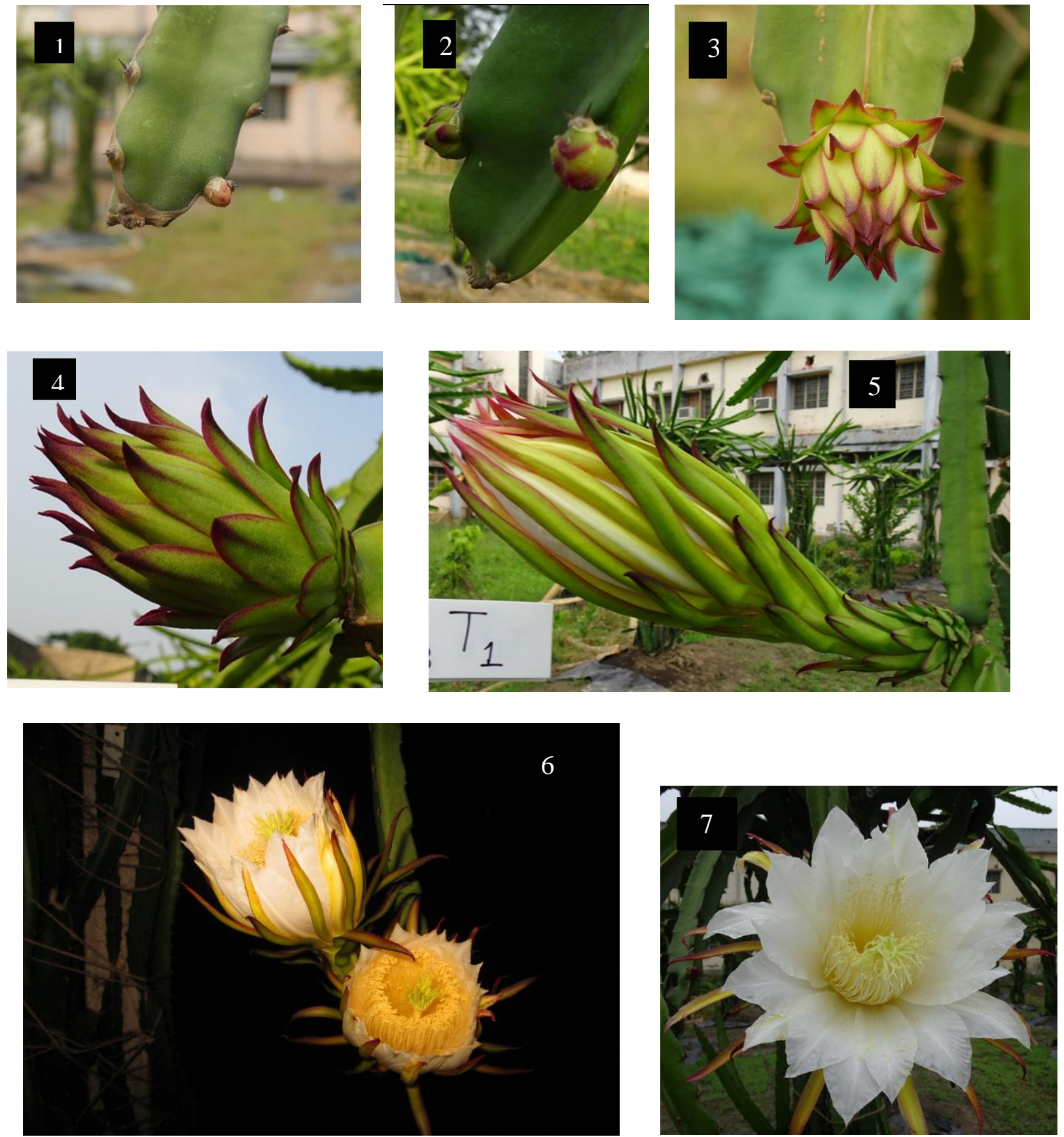

\section{References}

Britton N.L and Rose J.N. Descriptions and Illustrations of Plants of the Cactus Family, Vol. I and II, Dover Publ., Inc., New York, USA, 1963, pp. 183-195.
Luders, L. (1999). The pitaya or dragon fruit. Prim. Ind. Fish. North. Territ. Aust., pp. 778.

Mizrahi, Y., Nerd, A. and Nobel, P. S. (1997). Cacti as a crop. Hort. Rev., 18: 291-320. 
Mizrahi. Y and Nerd. A (1999). Climbing and columnar cacti- new arid lands fruit crops In Janick J ed Perspective in new crops and new crops uses, ASHS Press, Alexandria, USA, 358-366.

Morton, J. Cactaceae: strawberry pear and related species. In: Fruits of Warm Climates, Ed., Miami, and Fl. 1987, pp. 347-348.

Ortiz-Hernández

HYD

(1999).

Pitahayaunnuevo cultivopara México Ed Limusa-Grupo Noriega Editors México DF Mexico, 111.

Perween, T. Mandal, K.K and Hasan, M.A. (2018). Dragon fruit: An exotic super future fruit of India. J. Pharm. and Phytochem, 2018, 7(2): 1022-1026.

Daubresse Balayer, M. Le pitahaya, Fruits Oubliés, 1999, 1: 15-17.

\section{How to cite this article:}

Tamanna Perween and Hasan, M.A. 2018. Effect of Different Dose of NPK on Flower Phenology of Dragon Fruit. Int.J.Curr.Microbiol.App.Sci. 7(05): 2189-2194.

doi: https://doi.org/10.20546/ijcmas.2018.705.255 